\title{
Las distancias sociopsicológicas de la migración española e italiana cualificada en México
}

\section{The sociopsychological distances of skilled Spanish and Italian migrants in Mexico}

\author{
Xavier Oliveras González \\ Departamento de Estudios Urbanos y Medio Ambiente \\ El Colegio de la Frontera Norte, Sede Matamoros, Tamaulipas, México \\ orCiD: http://orcid.org/oooo-0002-1712-3459 \\ DoI: http://dx.doi.org/ı.28928/revistaiztapalapa/8420I8/atc4/oliverasgonzalezx
}

\begin{abstract}
Resumen
Se analiza la dimensión sociopsicológica de los procesos migratorios a partir de las representaciones espaciales y sociales (re)producidas sobre el lugar de destino antes y después del establecimiento allí. Para ello se aplicó una metodología cualitativa: se realizaron entrevistas semiestructuradas a inmigrantes de alta cualificación establecidos en México, originarios de España e Italia. La interpretación de los datos se realizó desde el enfoque teórico de las distancias sociopsicológicas (cognitiva, afectiva y mental). A diferencia de la hipótesis de partida, se encontró que el proceso migratorio supone para algunos informantes una reelaboración de las representaciones de acuerdo con la nueva realidad experimentada (reducción de las distancias), mientras que para otros permanecen las representaciones anteriores e incluso se refuerzan (permanencia de las distancias).

Palabras clave: migración cualificada; distancias sociopsicológicas; representaciones; incorporación social de inmigrantes; México.
\end{abstract}

\footnotetext{
Abstract

It is analyzed the sociopsychological dimension of the migration processes in relation to the (re)production of spatial and social representations about the place of destination before and after arriving there. That objective was achieved through a qualitative methodology: it was applied a semi-structured interview to immigrants from Spain and Italy living in Mexico. Data was interpreted through the theoretical approach of sociopsycological distances (cognitive, affective, and mental distances). Differing from the initial hypothesis, findings show that for some informants migration implied a redefinition of the representations according to the new experienced reality (a reduction of the distances); however, for others, the previous ones remained and even were bolstered (a permanence of the distances). Key words: skilled migration; sociopsychological distances; representations; immigrant social incorporation; Mexico.
}

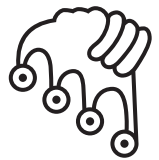

IZTAPALAPA

Agua sobre lajas

* xoliveras@colef.mx 
Cegún una nota publicada en un medio digital mexicano y compartida amplia$\int$ mente en las redes sociales, el conocido artista español Salvador Dalí habría dicho en algún momento que "De ninguna manera volveré a México. No soporto estar en un país más surrealista que mis pinturas" (Cuautle, 20I4). En espacios como el grupo Facebook "Españoles en México", integrado por migrantes españoles en el país y usuarios de nacionalidad mexicana, la afirmación generó un centenar largo de comentarios y réplicas. En medio de un creciente número de insultos y groserías, se debatió sobre lo que es México; es decir, quienes participaron en el debate expresaron y reprodujeron las ideas y estereotipos más habituales sobre este país. Esto lleva a señalar que, precisamente, uno de los múltiples factores de los que depende la decisión de migrar, la inserción laboral y la integración en el país y ciudad de destino es el conjunto de creencias, ideas, valoraciones, prejuicios, estereotipos o aspiraciones en un momento y contexto dados, sobre el lugar y sociedad de destino y sobre sus actividades; es decir, de las "representaciones sociales".

El presente artículo tiene por objetivo explorar las distancias sociopsicológicas del proceso migratorio mediante la identificación y comparación de las representaciones que sobre México la población migrante (re)produce antes y después de su establecimiento en el aquel país. Las representaciones son, en este sentido, consideradas como indicadores de aquellas distancias. Los resultados son fruto de un proyecto de investigación cualitativa en el que se entrevistaron inmigrantes de alta cualificación originarios de España e Italia establecidos recientemente en México. La migración a este país, en tanto que receptor, ha sido escasamente estudiada, y mucho menos aún para el caso de la migración cualificada; en este sentido, el artículo quiere ser una contribución a este campo. Los colectivos estudiados están asentados en las principales áreas metropolitanas del país (Ciudad de México, Monterrey, Puebla y Guadalajara), a la par entre las más globales y, por lo tanto, centros de atracción de flujos de capitales, bienes, información y personas. El proyecto buscó identificar las motivaciones, la trayectoria y movilidad laborales, la construcción de redes, las cotidianidades y las formas de dar sentido en el nuevo país. 
Para la identificación e interpretación de las representaciones se ha utilizado el enfoque teórico de las llamadas distancias sociopsicológicas (van Houtum, 2000; Karakayali, 2009). Brevemente, la hipótesis de partida relaciona las representaciones con el grado de familiaridad de un individuo y/o un colectivo con un espacio y una sociedad: a menor familiaridad (mayor distancia) menos se conoce, entiende o aprecia el espacio y sociedad, lo que lleva a la formación de simplificaciones, prejuicios y estereotipos; $y$, al contrario, a mayor cercanía, más se conoce, entiende y aprecia. En este contexto, la evaluación de los cambios (las diferencias y semejanzas) entre las representaciones anteriores y posteriores al establecimiento en México permite evaluar si ha aumentado la aprehensión de la realidad, la conexión afectiva y la comprensión de las convenciones y, por lo tanto, si se reducen, matizan o complejizan los estereotipos, prejuicios, valoraciones, etc. Ello constituye un indicador de la integración de los migrantes en el país de residencia y, sobre todo, de la apropiación y adaptación de las representaciones sobre México (re)producidas por las poblaciones locales (es decir, sobre sí mismas y sobre su país).

Las representaciones relativas a México han sido ampliamente abordadas desde las ciencias sociales y para distintos ámbitos (el turismo, la literatura, la cultura popular, la educación escolar, los medios de comunicación, el arte, etc.) (Zúñiga, 1998; Rajchenberg y Héau-Lambert, 2005; Cota, 2007; Giménez, 2007; Olmos, 20II; Méndez y Velázquez, 2013; entre muchos otros). Igualmente son numerosos los análisis de las representaciones sobre este país (re)producidas en el extranjero y por extranjeros, de las que destacan las construidas en los Estados Unidos y su relación con la política, el derecho, la economía o la cultura en aquel país (Peña, I998; Bender, 20I2; San Miguel, 20I6). En cambio, las representaciones de los colectivos extranjeros / migrantes en México han recibido una menor atención (Ortiz y Mendoza, 2008; Yarto, 20II). Su análisis desde las distancias sociopsicológicas constituye una novedad, ya que este enfoque se ha aplicado particularmente en individuos y/o colectivos caracterizados por la inmovilidad (o ausencia de desplazamiento) y, en menor medida, por un desplazamiento temporal (como el visitante y el turista) (van der Velde y van Naerssen, 20II). En esta dirección, la condición de migrante, derivada del desplazamiento y permanencia en un espacio distinto al de origen, ofrece la oportunidad de evaluar los cambios en las representaciones entre distintas etapas vitales. La diferencia entre el migrante y los otros perfiles no solo reside en la duración de la permanencia en un espacio distinto al de origen, sino que también hipotéticamente en un acercamiento sociopsicológico, además del físico geográfico.

Por último, el presente artículo se inicia con la aproximación teórica de las distancias sociopsicológicas (cognitiva, afectiva y mental), seguido de la presentación de la 
metodología utilizada para el análisis y sus alcances y limitaciones. A continuación, se exponen los resultados obtenidos para la población española e italiana residente en México, divididos en dos partes, las representaciones anteriores y posteriores a su residencia en este país. Finalmente, se cierra con unas conclusiones.

\section{Las representaciones sociales}

El concepto de "representación social", así como el de "imaginario", con el que a menudo erróneamente se intercambia, es de difícil definición y las teorías que lo abordan son heterogéneas y con escasos consensos. Su amplio uso en las ciencias sociales es indisociable de la dificultad de marcar unos límites precisos entre los procesos psicológicos y socioculturales que intervienen en su formación. Para esta investigación se parte, de manera muy esquemática, del campo teórico-analítico desarrollado por Serge Moscovici y profundizado por Denise Jodelet, según el cual las "representaciones sociales" son un corpus organizado de conocimientos gracias al cual los individuos hacen inteligible la realidad física y social, y se integran en un grupo o una relación cotidiana de intercambios. Los procesos psicológicos y socioculturales, entre los cuales existe una relación de mutua influencia (Jodelet, 1984; Peña, 1998), contribuyen a la interpretación y comprensión que los individuos dan a la "realidad" (aunque la "realidad" no es algo definido ni acabado), al mundo exterior a sí mismos; es decir, en relación con los otros y con el entorno. Así, las representaciones sociales están conformadas por las creencias, imágenes, valoraciones, estereotipos, prejuicios, ideas, conceptos y convicciones "que se definen en torno a una actividad, un espacio, un periodo o una persona (o una sociedad) en un momento dado" (Hiernaux, 2002: 8) y, por lo tanto, son variables, cambiantes, coyunturales y en permanente remodelación.

Las representaciones, en tanto que un corpus organizado de distintos y variados referentes, están sujetas a los conocimientos, experiencias, creencias y emociones (re)producidas en el contexto psicológico, social, cultural y espacial en el que se desenvuelven los individuos. Desde la geografía, por ejemplo, la inclusión y abordaje de las representaciones, así como de los imaginarios, contribuye a la comprensión de la relación de las sociedades con el espacio, el propio y el ajeno (Lindón y Hiernaux, 20I2). Así, por ejemplo, las representaciones incluyen una significativa cantidad de referentes espaciales, organizados en estructuras metageográficas verosímiles con el objetivo de dar sentido, orientarse, comunicar, explicar, etc. el mundo, el propio, el cercano y el lejano (Lewis y Wigen, 1997). Igualmente, en ámbitos interculturales, 
como el de las migraciones internacionales, pero también el turismo y las relaciones internacionales, la relevancia de las representaciones es también objeto de interés. Los individuos que viven, experimentan e interactúan con individuos, sociedades y espacios de otros ámbitos socioculturales lo hacen con una representación previa que media aquella interacción (Zúñiga, 1992; Goycoechea y Ramírez, 2002; García y Verdú, 2008; Devadason, 20I0).

\section{Distancias sociopsicológicas}

Se propone interpretar y analizar las representaciones a partir del enfoque de las distancias sociopsicológicas (van Houtum, 1999, 2000; Karakayali, 2009; Tasci, 2009). Esta aproximación teórica parte de tres asunciones: primera, que la experiencia de la realidad y la "realidad" no son lo mismo ni que una y otra son congruentes; segunda, que el conocimiento y comprensión de la "realidad", es decir, hacerla inteligible, se obtiene a través de la conexión y experimentación con aquélla, y no con el distanciamiento; $y$, tercera, que los diferentes grados de conocimiento, comprensión y afectividad median las relaciones interpersonales y sociales. De todo ello se deriva que las características que toman las relaciones de un individuo con su entorno están determinadas por la cercanía o lejanía a la "realidad" y que aquéllas contribuyen a la diferenciación y separación entre lo interno / lo propio y lo externo / lo ajeno; entre "nosotros" y "ellos" / los "otros". Asimismo, las distancias sociopsicológicas (al igual que las representaciones) no son absolutas ni objetivas, por el contrario, son relativas y subjetivas, dependientes de cada individuo ( $y$ por lo tanto de su etapa vital, experiencias, formación, relaciones interpersonales, etc.) y colectivo. Son, por lo tanto, un fenómeno sociocultural potencialmente relevante en la formación y reproducción de representaciones.

A partir de esta concepción general, diferentes estudios señalan la existencia de varias distancias sociopsicológicas (de ahí el uso del plural), basada cada una de ellas en distintas concepciones y enfoques, aunque en determinados casos se solapan. Sirvan de ejemplo las síntesis elaboradas por van Houtum (2000), que las clasifica en tres tipos (cognitiva, afectiva y mental), y Karakayali (2009), en cuatro (afectiva, normativa, interactiva y cultural). Como refleja la elección del término “distancia", en origen su conceptualización estaba directamente relacionada con enfoques metodológicos cuantitativos, en tanto que categorías medibles (por medio de test, incluida la elaboración de mapas, dibujos, estimaciones, etc.); por el contrario, ha sido menos frecuente y más reciente su abordaje desde metodologías cualitativas. Por otra parte, 
si bien su desarrollo inicial se dio en el ámbito de la psicología en los años 1920-1940, tanto teórica como metodológicamente fue adoptado posteriormente, a partir de los años 1960, por otras disciplinas de las ciencias sociales. Este es el caso de la geografía, en estudios sobre percepción y valoración del espacio (destinos turísticos, regiones fronterizas, movilidad, etc.) (Ankomah, Crompton y Baker, 1996; van Houtum, 1999; Tasci, 2009); de la sociología, en estudios de identidad, afectividad e interrelaciones grupales y sociales (Peña, 1998; Karakayali, 2009; Cárdaba y Brändle, 2013); y de las ciencias de la administración y de la comunicación, en estudios sobre innovación, colaboración, transferencia y comunicación (Nooteboom, 2000; Amin y Roberts, 2008; Muscio y Pozzali, 2013).

Para los objetivos de la investigación se parte de la clasificación de van Houtum (2000), cuyas tres categorías se definen por una correlación entre la distancia y la familiaridad. En primer lugar, la distancia cognitiva se define como la diferencia entre la representación / percepción de la realidad y la "realidad" (un espacio, una actividad, una sociedad, etc.), donde la primera está basada en el conocimiento y la experiencia de aquélla. En este caso, cuanto más se conoce y se experimenta un espacio (mayor es la familiaridad), menor es la distancia cognitiva. Un indicador comúnmente utilizado para determinar aquella distancia es la desviación entre longitudes y superficies reales y estimadas. Por ejemplo, en la localización y dimensiones de un lugar y en la distancia entre dos lugares. En segundo lugar, la distancia afectiva se define como el grado de conexión emocional de un individuo con un espacio o una sociedad, basándose en la idea que el espacio, sociedad o individuo que está más cercano a uno es el que se siente emocionalmente más cercano (menor es la distancia). En este caso, uno de los indicadores habituales es la (re)producción de estereotipos, prejuicios e idealizaciones sobre el "otro" y lo "otro", entendiendo que, a mayor ausencia de conexión afectiva, mayor es la probabilidad de (re)producirlos. En tercer y último lugar, la distancia mental se refiere a las diferencias en las convenciones sociales, culturales, lingüísticas, normativas o económicas entre un espacio y/o sociedad y otro. En este caso, a mayor distancia, mayor es la incomprensión, la incertidumbre y la desconfianza, y su reducción requiere un proceso de aprendizaje, que lleva a la comprensión y hasta la adopción (van Houtum, 1999; Karakayali, 2009).

Teniendo en cuenta que la realidad y las representaciones son relativas y subjetivas a cada individuo (en un contexto social dado), el planteamiento de una reducción o incremento absoluto de las distancias no tiene sentido, ya que no hay un límite o punto final esencial a los que llegar; el acercamiento / alejamiento es, por lo tanto, infinito (Lussault, 2015). Sin embargo, no por ello deja de haber factores que contribuyen a la reducción, incremento o mantenimiento (relativo) de las dis- 
tancias. Pueden señalarse al menos tres: la movilidad / inmovilidad, la difusión y comunicación, y la imitación. En relación al primer factor, el desplazamiento y la convivencia en un espacio ajeno aumenta la probabilidad de verificar, matizar o modificar las representaciones sobre aquél; por el contrario, en un contexto de inmovilidad las representaciones tienden a ser persistentes, ya que virtualmente no hay posibilidad de incrementar la aprensión de la realidad, la conexión afectiva o la comprensión de las convenciones. En segundo lugar, a través de los medios de difusión y comunicación, como son el cine, la literatura, la prensa y las tecnologías de la información y comunicación, un individuo puede experimentar un espacio ajeno sin estar físicamente allí. En este sentido, la aprehensión, conexión y comprensión en ausencia de una copresencia puede, aunque solo en cierta medida, sustituir las limitaciones de la inmovilidad, lo que a su vez permite construir una determinada coespacialidad. Por último, la imitación implica que, independientemente de si existe una copresencia o no, un individuo o colectivo adopta y adapta las representaciones de los otros, debido a su prestigio, influencia, dominio, etc. A pesar de lo señalado, como se ha demostrado en varias investigaciones (Cárdaba y Brändle, 2013), el acercamiento que permite tanto la movilidad como la difusión y comunicación (la "hipótesis del contacto") no equivale necesariamente a una reducción de las distancias sociopsicológicas; al contrario, pueden permanecer, reforzarse o incluso incrementarse, especialmente en el caso de las representaciones negativas (los estereotipos y prejuicios), ya que tienden a autoperpetuarse en el tiempo y son realmente resistentes al cambio.

Por último, la reducción de las distancias sociopsicológicas en el caso de los individuos y colectivos migrantes, que conlleva por definición el desplazamiento y convivencia en un espacio ajeno (al menos así asumido al inicio), deviene un proceso de evolución y transitoriedad desde la observación de lo ajeno hacia su adaptación y/o adopción, y mediante el cual progresivamente se incrementan la aprensión de la realidad, la conexión afectiva y la comprensión de las convenciones. Para este proceso, si bien puede fijarse un punto de inicio (el momento de tomar la decisión de migrar o de la llegada al lugar de destino), difícilmente puede determinarse su conclusión en tanto que no existe una realidad esencial a la que llegar; por ello este proceso se caracteriza por su permanente transición. Siguiendo a Anzaldúa (1987, 1993), en este sentido se puede decir que la población migrante se encuentra simultáneamente "afuera" y "adentro" y se mueve en un estado sociopsicológico intermedio (in-between), ambivalente, transitorio e híbrido entre las representaciones del espacio / sociedad de origen (lo que les es propio) y la del de destino (lo que les es 
ajeno, pero que a la vez van conociendo), donde los referentes se van renegociando y articulando una y otra vez.

\section{Metodología}

Como se ha señalado en el apartado anterior, si bien las distancias sociopsicológicas han sido abordadas habitualmente desde un enfoque cuantitativo, en este caso para identificar las características de las representaciones y los cambios entre las anteriores y posteriores a la migración se optó por uno cualitativo. Se aplicaron en concreto dos métodos: una entrevista semi-estructurada con guión y un análisis de contenidos a la información recabada.

En primer lugar, la entrevista se aplicó a un muestreo no probabilístico de españoles e italianos residentes en México hasta alcanzar un punto de saturación. Con este tipo de muestra se consigue informar de cómo es el universo, aunque no permite saber con qué precisión. Para la selección de los informantes se estableció un conjunto de criterios: ser españoles o italianos por nacimiento, llevar viviendo en México al menos 6 meses y haber inmigrado al país después de sus estudios superiores y/o formación profesional; además se buscó en la medida de lo posible un equilibrio en la nacionalidad, el sexo y las categorías profesionales y/o laborales. Dado que se trataba de estudiar dos colectivos muy específicos, con poblaciones de baja incidencia e individuos de difícil acceso, para la obtención de la muestra (y la concertación de entrevistas) se combinaron tres técnicas: contactos previos con informantes (en caso de existir), contacto a través de las redes sociales (grupos Facebook de españoles y de italianos en México) y de la técnica conocida como bola de nieve (informantes conocidos por aquellos previamente entrevistados). Las entrevistas se llevaron a cabo en 2014 y 2015, y se realizaron un total de 129: 4I en Ciudad de México, 3I en Monterrey, 3I en Guadalajara y 26 en Puebla; 8I hombres y 48 mujeres; y 106 nacidos en España y 23 nacidos en Italia. La entrevista está organizada en cuatro apartados temáticos: trayectoria migratoria, perspectiva laboral, perspectiva social y perspectiva geográfica. No es necesario describir cada uno de ellos, solo indicar que en el segundo y cuarto se incluyeron preguntas explícitamente relacionadas con las representaciones. En total fueron cinco, tanto en relación con las representaciones anteriores como posteriores a la llegada a México: en primer lugar, “¿Qué conocía de México antes de llegar? ¿Cómo se lo imaginaba antes de llegar?”, y en segundo lugar "¿Qué imagen tenía del país y de la ciudad de residencia a su llegada?, ¿Ha cambiado desde que usted reside aquí? y ¿En qué sentido?”. 
Para el análisis de los resultados y de ellos extraer conclusiones válidas es necesario tener en cuenta algunos alcances metodológicos. En primer lugar, dado tanto las características de la entrevista como la función y estructura del corpus que forma las representaciones, los entrevistados informaban directa o indirectamente sobre aquéllas en las respuestas a otras preguntas, ante lo cual el entrevistador debía considerar la necesidad o no de formular las preguntas diseñadas y/o modificarlas. Por otra parte, considerando que el grueso de preguntas relativas a las representaciones se planteaba en el último apartado, momento en el cual entrevistado y/o entrevistador podían estar más fatigados, así como sufrir por premuras de tiempo (larga duración de la entrevista, horarios restringidos del entrevistado, etc.), es probable que en varios casos las respuestas contuvieran menos información que si las condiciones hubieran sido otras. En tercer lugar, no se trata de un estudio longitudinal, en el que se entrevistara a los informantes tanto antes como después de su migración, sino que solo fueron entrevistados una vez estaban residiendo en México, es decir, una vez habían devenido migrantes, en el sentido sociológico del fenómeno migratorio (o "inmigrantes", "exiliados" o "expatriados" como ellos se autoidentificaron). Es decir, las representaciones anteriores no fueron expresadas con anterioridad al proceso migratorio ni a la construcción de una "nueva" identidad, sino que las respuestas fueron mediadas y reinterpretadas a partir del recuerdo y la experiencia en el país de destino. Para reducir o anular esta limitación debería conducirse un estudio longitudinal, aunque realizar entrevistas previamente a la inmigración resulta poco factible.

En segundo lugar, la información recabada fue posteriormente analizada mediante un análisis de contenido. Este método permite formular inferencias reproducibles y válidas, y su aplicación implica considerar tres aspectos: los datos tal y como fueron comunicados al entrevistador (la transcripción de las entrevistas); el contexto de los datos (por ejemplo, la fatiga y los horarios), y la subjetividad y conocimientos del investigador. Su aplicación requiere tres etapas básicas, la simplificación (reducción de la información bajo el criterio de la relevancia interpretativa, y selección sólo de los datos pertinentes y relevantes relativos para los objetivos de la investigación), la categorización (clasificación de los datos a partir de un conjunto de criterios previamente definidos a partir de lo cual se elabora un sistema de categorías), y la redacción de los resultados.

De acuerdo a los objetivos de la investigación las categorías se organizaron en dos grupos: representaciones sobre México antes de inmigrar, y representaciones sobre México después de inmigrar. Con relación al primer grupo, se identificaron y definieron tres categorías principales: a) ausencia y/o presencia de representaciones 
(o no se recuerda o se esconde); b) referentes de las representaciones; y c) fuentes de los referentes. Para el segundo grupo: a) verificación de la totalidad o parte de los referentes de las representaciones; b) reafirmación y/o matización de la totalidad o parte de los referentes; c) identificación de nuevos referentes; $y$ d) reconocimiento de un desconocimiento o conocimiento parcial, impreciso o incompleto previos. Por último, la estructura de la redacción de los resultados deriva de aquella categorización, a la vez que se basa en una descripción emic donde se refleja el punto de vista de los informantes en términos significativos (conscientes o inconscientes), por lo que se incluyen referencias a los datos cualitativos recopilados (indicados con el pseudónimo asignado a los informantes, la nacionalidad, la ciudad de residencia y el año en el que migraron a México).

Por último, y regresando a la subjetividad y conocimientos del investigador, resulta obligatorio indicar su relación con el espacio y colectivo de estudio, ya que la aplicación de la metodología e interpretación de los resultados están marcadas por su experiencia como individuo; en este caso el investigador entra también en la definición de inmigrante español altamente cualificado en México. Ello tiene la ventaja que investigador e informantes comparten en mayor grado el contexto y las estructuras sociopsicológicas, lo cual facilita la identificación y comprensión de las representaciones de los informantes (ver, por ejemplo, Ortiz y Mendoza, 2008); por el contrario, un investigador mexicano comparte las representaciones (re)producidas por la sociedad mexicana, que facilita la evaluación del grado de adopción y adaptación de las representaciones mexicanas por parte de los informantes (ver, por ejemplo, Yarto, 20II).

\section{Algunos hallazgos}

El análisis de contenido permite diferenciar las representaciones sociales de los sujetos de estudio asociadas a México antes de su llegada y las representaciones sociales elaboradas a partir de la experiencia de vida local.

\section{Representaciones asociadas a México (anterioridad a la migración a México)}

El análisis de las entrevistas permite la identificación de tres representaciones principales asociadas a México por parte de españoles e italianos. Todas ellas reflejan a la vez un espacio ajeno (México) y un espacio propio (los países y sociedades de 
origen, España e Italia). El primero aparece a menudo mediante los estereotipos y los prejuicios, mientras que el segundo a través de la comparación, presente aunque no esté explícitamente mencionado (México es lo que no es España / Italia). Uno y otro espacio son indicativos de una construcción y comunicación de las representaciones en el marco de unas amplias distancias sociopsicológicas, donde lo desconocido e incomprendido es interpretado a partir de referentes propios.

La primera representación corresponde a lo que para algunos informantes constituía un "espacio en blanco", del que afirman no tener (o no recordar) ninguna idea en específico o tener solamente algunas nociones vagas, dispersas e incoherentes. Sirvan de ejemplo las palabras de Josefina (española, Ciudad México, 2007), quien respondía que "no tenía una imagen clara del país". Esta representación denota unas distancias sociopsicológicas extremas, donde la ausencia de familiaridad es tal que el ente espacial y social "México" prácticamente se reduce a la mera existencia del nombre. En este caso, los informantes que comparten esta representación en ocasiones corresponden con quienes llevan más tiempo en el país y, por lo tanto, con una mayor probabilidad de haber olvidado $y / o$ de esconder conscientemente el pasado (quizá por vergüenza); en otros casos, sin embargo, se trata de migrantes más recientes.

En segundo lugar, México era identificado como "un país idealizado y romantizado", en un sentido muy positivo, que a la vez denota determinados referentes realistas sobre los países de origen. Bajo esta representación se agrupan aquellas ideas relativas a un México "auténtico" y "tradicional", al patrimonio prehispánico y colonial, a un país tranquilo y feliz, etc. A diferencia del anterior, este corpus está plenamente articulado y las distancias sociopsicológicas son menores, especialmente en cuanto a la distancia afectiva. Un ejemplo de ello lo ofrecen Juan Manuel (español, Monterrey, 2008) y Carla (española, Puebla, 2010), para quienes México se asociaba a:

un país del mariachi, de los boleros, del picante, de la facilidad del mexicano de ser así un poco vago;

los típicos estereotipos del cactus y el tequila, y el sombrero, sentado en la puerta con un burro al lado, y $[\ldots]$ de que era muy bonito, que la gente era muy amable y el ambiente cálido de la gente

Estos dos fragmentos muestran, además, que la comunicación de las representaciones por los informantes durante la entrevista se basaba en una reflexión posterior a la llegada al país. Así, el gusto por la comida picosa, los mariachis, la amabilidad y 
calidez de la población mexicana, y el mexicano echándose una siesta recostado bajo la sombra de un nopal ya son identificados como estereotipos habituales asociados al país. Sobre ello se ampliará en el siguiente apartado.

La tercer y última representación es la antítesis de la anterior, el de un país menos desarrollado, más pobre y más inseguro. Mientras que en aquélla el pasado, la ruralidad y la pereza eran valorados positivamente, en ésta al contrario se valoran negativamente. Para los informantes aquellos referentes vienen a significar un retraso social, económico e incluso moral respecto a los países de origen. Puesto que para varios de los informantes el motivo de la migración fue laboral, sus respuestas denotaban una especial preocupación por la pereza e incompetencia del trabajador mexicano, reproduciendo así uno de los principales estereotipos y prejuicios relativos a México. Asimismo, aquel menor desarrollo y pobreza están asociados a la inseguridad, la violencia, la corrupción y la impunidad. Como en los casos anteriores, algunos fragmentos lo ilustran (que también deben tomarse con precaución al haber sido reconstruidos con posterioridad al proceso migratorio); por ejemplo, Camilo (español, Monterrey, 20ıо) "pensaba que era un país en vías de desarrollo, más acercado al tercer mundo", mientras que para Tadeo (español, Guadalajara, 20I2) "la imagen que tenía [era] de inseguridad, mucha violencia, muchos robos, mucha corrupción". A causa de estos referentes negativos, distintos entrevistados afirman que habían sentido un determinado grado de miedo al momento de decidir migrar al país y/o elegir una ciudad y una colonia donde residir. Así, por ejemplo, Pepe (español, Guadalajara, 20I2) admite:

entonces sí tenía inseguridad de venirme y sí, un poco, no de miedo, pero sí de intranquilidad. Digo, no voy a poder salir a caminar solo o no voy a poder ir en el coche.

Un referente común y compartido en las tres representaciones son las estimaciones más o menos erróneas de la localización y la dimensión de México, lo que constituye un claro indicador de una significativa distancia cognitiva. Para varios entrevistados "México" no está en o no es un país de "América del Norte", sino en o de "América del Sur". Esta idea se apoya frecuentemente en la estructura metageográfica que asocia "América del Norte" con las representaciones del Primer Mundo, los países desarrollados y los países de lengua inglesa, mientras que "América del Sur" lo es con el Tercer Mundo, a los países subdesarrollados y a los países de lengua latina (Lewis y Wigen, 1997). Reflejo de ello son las respuestas de Llorenç (español, Monterrey, 200I) y Elena (española, Guadalajara, 200I): 
se piensa que México está en Sudamérica. [... ] hay una América del Norte que se compone de Estados Unidos y Canadá, y una América del Sur que se compone de todo lo demás.

Ni siquiera sabía que [México] estaba en Norteamérica. Yo me peleé el primer año cuando me regresé a España explicándole a la gente, tuve que sacar un mapa y demostrarles físicamente que México está en Norteamérica, no en Sudamérica.

Asimismo, varios informantes estiman un país mucho más grande o mucho más pequeño (en superficie) de lo que es, tal y como César (español, Guadalajara, 2009) muestra al indicar que México es "un país grande, pero no me imaginaba tanto. Colocar México así es como Europa... No te das cuenta que Los Cabos es como Italia". Este fragmento muestra además dos operaciones frecuentes en casos de lagunas cognitivas, la sustitución de una parte por el todo (en este caso, Los Cabos por la península de Baja California) y la comparación con un referente propio (en este caso, Europa e Italia) ${ }^{1}$.

Por último, a partir de las entrevistas se pueden identificar seis fuentes principales de los referentes de las representaciones. Cinco de ellas están directamente relacionadas con la difusión y adquisición de ideas, imágenes, valoraciones, etc. en un marco de inmovilidad, lo cual concuerda con una etapa de vida previa a la migración, mientras que la sexta fuente se da en un marco de movilidad temporal vinculada al turismo.

Sin que pueda asegurarse con exactitud cuáles son las fuentes más comunes de los referentes positivos (el México tradicional, alegre, feliz, etc.), éstas parecen ser los documentales, revistas y páginas web de viajes sobre México, así como el cine, la televisión, la música y el arte. En esta dirección, Maria (italiana, Monterrey, 2013) comenta que "te imaginas el México que te pasan en el National Geographic". Cabe indicar que el origen de las fuentes en ocasiones está en México y en otras fuera de este país, notablemente en los Estados Unidos (como en el caso de National Geographic), o una combinación de ambas. De ello es indicativo las palabras de Christian (español, Ciudad de México, 1999), cuando dice que "No tenía una imagen del país, aparte de la imagen Speedy González, [... ] y de Cantinflas”. Para los referentes negativos (pobreza, inseguridad, violencia, etc.) las fuentes más comunes

1 La comparación es bastante exacta: mientras que la península de Baja California tiene una longitud de I,247 km (de Mexicali a Cabo San Lucas), la de la península Itálica es de unos I,00o km (entre el valle del Po, al norte, y Calabria, al sur). 
suelen ser los medios de comunicación, así como también el cine y la televisión. Sirva de ejemplo el siguiente fragmento extraído de la entrevista a Pepe (español, Guadalajara, 20I2), para quien, "La imagen que tenía del país era un desastre. Por desgracia, todas las noticias que llegan a Europa son siempre negativas".

Entre los informantes que compartían una representación de México como un "espacio en blanco", el proceso de decisión de migrar a México se acompañaba de una búsqueda y consulta de información, de la que resultan habituales dos fuentes, tanto para referentes positivos como negativos. De un lado, amigos, parientes y conocidos que hubieran estado previamente en el país por motivos de turismo y/o por trabajo, y/o que eran de origen mexicano, y del otro, fuentes de información que consideraran confiables, como las embajadas de los países de origen en México y las enciclopedias. De esta última es representativo lo expresado por Jaime (español, Puebla, 1999):

Te metes a la página [web] de la embajada española o del ministerio de asuntos exteriores, te pone ahí que es un país en desarrollo, con un cierto grado de inseguridad: vacúnate de tifoidea, vacúnate de no sé qué.

y por José (español, Guadalajara, 2008), quien "no sabía absolutamente nada más que lo que había leído en la Wikipedia antes de salir."

Por último, hay informantes que declaran que algunas de las ideas que tenían sobre México se derivaban de visitas anteriores al país. La mayoría de los viajes que habían realizado fueron por motivos de turismo, aunque en algún caso también por trabajo. Los referentes predominantes en este caso suelen ser positivos, asociados a los resorts del país, como son Los Cabos, antes mencionado, y Cancún, como comenta Olivia (italiana, Monterrey, 2002), según la cual "Para los europeos lo máximo de México, o su conocimiento de México es Cancún".

\section{Representaciones asociadas a México (posterioridad a la migración a México)}

A partir del análisis de contenidos se constata que, para varios entrevistados, las distancias sociopsicológicas se han reducido a partir de vivir, haber viajado a distintos lugares y/o integrarse en una red afectiva y de cuidados (familia, pareja, amigos) en México. Con relación a la distancia cognitiva, se detectan dos procesos básicos, simultáneos e integrados en los discursos de los informantes. En primer lugar, se hacen conscientes de que las ideas, valoraciones, estereotipos y prejuicios que tenían antes de instalarse en México no se ajustan con la experiencia de la realidad una vez 
en el país. En esta dirección, por ejemplo, se manifestaba claramente Juan Manuel (español, Monterrey, 2008), que afirmaba que "cuando estás aquí ves que México es un país que para nada corresponde al estereotipo". Esta toma de consciencia lleva en ocasiones a reconocer explícitamente que con anterioridad se tenía un desconocimiento o un conocimiento impreciso e imparcial de la "realidad" mexicana.

En segundo lugar, en paralelo a una creciente experiencia de la realidad, los migrantes construyen nuevas representaciones en las que agregan referentes más precisos o más ajustados a aquella realidad, ya sean positivos o negativos y que, como se señalará un poco más adelante, les permite confirmar o rechazar las representaciones anteriores. Así, en el lado positivo, México deviene un país trabajador, urbanizado, dinámico, en crecimiento y de oportunidades, un país comparable con o del Primer Mundo, y un país donde se puede vivir bien. Ese mayor desarrollo económico y urbano de lo imaginado previamente proporciona a varios migrantes seguridad y confort y una cercanía sociopsicológica con sus lugares de origen, aunque en ocasiones a algunos informantes les cuesta admitirlo. En el lado negativo, se incluyen referencias a un país de muchas diferencias sociales y socioeconómicas (de nuevo, en comparación con los países de origen), a un país con carencias, rezagos y desordenado, y a un país corrupto, poco ético, violento e inseguro. En esta dirección, son indicativas las palabras de Gonzalo (español, Ciudad de México, 20II), para quien México:

Es la Suavicrema. [...]. Es una representación clara de lo que es México. Es el surrealismo absoluto por la corrupción por detrás. [...]. Cierta modernidad con corrupción y el absurdo completo.

Este fragmento ilustra además que la construcción y la comunicación de los referentes de las representaciones ya no se realizan partiendo del país de origen sino del de residencia. En este caso concreto, la idea de un México inseguro y corrupto es representada por "la Suavicrema", nombre popular con el que localmente se conoce el edificio Estela de Luz, en Ciudad de México, sobre el que se difundieron varios presuntos malos manejos en el presupuesto de su construcción e inauguración (Vargas, 2016). Esta es una referencia comprensible para la población de México y/o de Ciudad de México, y probablemente incomprensible por la población en el país de origen del informante; es decir, la distancia cognitiva del migrante se ha reducido respecto a la población local, mientras que se ha incrementado con relación a la sociedad de origen. 
Paralelamente a la reducción de la distancia cognitiva, también se detecta una amplia reducción de la distancia afectiva. En general, para los migrantes las conexiones afectivas aumentan a partir de experiencias satisfactorias: en las entrevistas manifestaron experiencias relativas a las oportunidades laborales (mejores puestos de trabajo, ascensos, realización profesional, etc.), la formación de una red afectiva y de cuidados (tener amigos, pareja o hijos), la vivienda (más grande y espaciosa que la que pudieran haber tenido en sus países de origen, por ejemplo) y las oportunidades de viajar y disfrutar del país (turismo, paisajes, gastronomía, arte, etc.). De ello son muestra los comentarios de Pietro (italiano, Monterrey, 2004), para quien "un sentimiento como el de vivir en México no lo he sentido tan fuerte [como en otros lugares]", y de Roberto (español, Ciudad de México, 200I), que decía que "me enamoré de México, sigo estando enamorado".

Con relación a la distancia mental, en tercer lugar, las entrevistas ponen de manifiesto que los migrantes progresivamente van comprendiendo y asumiendo las convenciones y normativas sociales, culturales, institucionales, económicas, etc. del país y/o de las poblaciones locales donde se establecen. Entre los referentes más frecuentemente mencionados están los relativos al comportamiento y proceder del trabajador, prestador de servicios o funcionario mexicano. Entre otros aspectos, algunos españoles e italianos subrayaban que aquél es incapaz de responder con un "no" a una petición que no quiere realizar o atender, ante lo cual los migrantes se sienten, primero, engañados $y / o$ decepcionados, para más adelante aprender a identificar aquellas respuestas afirmativas que realmente son negativas, y finalmente, sacar provecho de aquel proceder. Llorenç (español, Monterrey, 200I) lo explica de la siguiente forma:

Creo que me tardé dos años en decodificar al mexicano. Hay una cosa: por ejemplo, nunca te dicen "no". Está muy difícil luchar contra eso, porque no sabes a qué atenerte y tienes que saber leer el "no" aunque no te lo digan. Eso es un arte que tienes que aprender con el tiempo. [...]. La gente tiene como un horror a decir "no" o a enfrentarse a algo. Entonces siempre te dicen "s'" aunque sea "no". Si tu realmente crees que es "sí" cuando no lo es, te acabas defraudando. Pero no es maldad ni nada, simplemente una costumbre. Pienso que les molesta. Pero el "no" si es, lo que pasa es que no te lo dicen. Tienes que aprender a entender el "no" de una manera diferente. Nadie te va a decir "no" directamente.

Un caso similar se da con relación a la comprensión del tiempo y el uso de la expresión "ahorita". 
Vinculado a las representaciones de carácter espacial sobre México, sirva de ejemplo la comprensión por parte de los migrantes residentes en Monterrey de las convenciones relativas a "el Norte" y la región cultural norteña, de acuerdo a su propia experiencia y a la adopción del imaginario colectivo regiomontano (de clase media y alta) (sobre estas representaciones, véase Peña, 1998; Solís, 2005; Prieto, 20II; Yarto, 20II; Cantú et al., 20I6). Este doble proceso es simultáneo y difícilmente separable uno del otro. Recuérdese que, antes de migrar, una de las representaciones sobre México era el de un espacio en blanco, que es aún más frecuente con relación a las ciudades y regiones del país. En este sentido, los migrantes en Monterrey descubren y/o les son descubiertas, en primer lugar, las particularidades locales y regionales de esta ciudad, del estado (Nuevo León), del Noreste y/o del Norte. Posteriormente, en segundo lugar, descubren y/o asumen que "México" no es un espacio absoluto, uniforme y homogéneo, sino que se caracteriza por diferencias regionales a nivel geográfico, social, económico, cultural, ideológico, etc. Sirvan las palabras de Jorge (español, Monterrey, 2003), según quien "no existe México, sino muchos Méxicos". En tercer lugar, uno de esos Méxicos es "el Norte", cuyo corpus está compuesto por referentes a una región desarrollada, consumista, moderna y urbanizada, pero a la vez y, por ello mismo, con muchas desigualdades sociales. Como la población local de Monterrey, los migrantes explican las características de esa "realidad" por la influencia y cercanía de Estados Unidos. Así, por ejemplo, Claudio (italiano, Monterrey, 2000) comenta sin matices que "no sabía que el norte era como los Estados Unidos".

En contraste, los informantes residentes en Monterrey asumen y/o identifican que los referentes a la pobreza, el menor desarrollo, la vida tranquila, la ruralidad, la tradición, etc. son propios de las regiones centro y sur de México. Es decir, se produce una permanencia de los estereotipos, pero relocalizados, en un proceso de reducción de la distancia mental respecto a la población local, pero de mantenimiento de aquélla respecto al resto del país. Indicativo del proceso de adopción de las representaciones regiomontanas es el hecho de que varios de los informantes que identifican las diferencias regionales no habían visitado el Centro y/o el Sur antes de la entrevista. Otros, sí, como en el caso de Caín (español, Monterrey, 2007), quien no encontró el México que se imaginaba hasta visitar el Centro: "cuando llegué a Ciudad de México, el México que me imaginaba era ése”. En esta dirección, varios informantes reafirman su preferencia por un "México auténtico" relocalizado. Maria (italiana, Monterrey, 2013) lo resume al decir que: "Un México del norte que es diferente del México del sur [...]. El centro es más característico, como que me hubiera gustado más vivir en Guadalajara, en ciudades más culturales en este sentido y como más características [de la mexicanidad]". 
Para terminar, y con relación a esta última idea, mientras que para algunos entrevistados la reducción de las distancias sociopsicológicas conlleva el rechazo o matización de las representaciones anteriores, para otros los estereotipos y prejuicios permanecen y se refuerzan con la realidad experimentada, tanto con relación a un país idealizado y romántico, como a un país menos desarrollado, más pobre, más inseguro. Sirvan de ejemplo de ambas posturas las palabras de Marcos (español, Monterrey, 20I2) y Dario (italiano, Ciudad de México, 20II) respectivamente:

sí es un país bello, hermoso, con una cultura fuerte, una gastronomía importante. Entonces, todo lo que sabía de él sí se ha cumplido: un país lindo para vivir y acogedor, de gente muy amable y educada.

[La imagen] No ha cambiado. Es un país muy bonito, vivido por gente equivocada. Es un país muy bonito que ha sido cuidado muy mal en años pasados. $Y$ la gente de ahorita no tiene la mentalidad para cambiarlo.

Ambos fragmentos muestran un acercamiento afectivo por parte de los informantes, que se refleja en el reconocimiento de México como un país bonito / bello. Simultáneamente, y sin suponer una contradicción, la permanencia y reafirmación de las representaciones anteriores puede indicar, como sugieren Cárdaba y Brändle (2013), una forma de mantener la diferencia o la separación entre el "yo" / "nosotros" y lo "otro", entre lo propio y lo ajeno; es decir, entre lo español / italiano y lo mexicano.

\section{Discusión y conclusiones}

Con este artículo se deseaba explorar la pertinencia del enfoque teórico de las distancias sociopsicológicas para el análisis del proceso migratorio (tomando las representaciones anteriores y posteriores como indicadores); es decir, en casos en los que se produce un desplazamiento permanente o prolongado, en contraste con su aplicación habitual en casos de inmovilidad y desplazamiento temporal. El caso de estudio, las representaciones de dos colectivos europeos de alta cualificación en México, muestra que este enfoque supone una vía interesante para continuar profundizando. Sintética y brevemente, los resultados obtenidos indican que antes de migrar los informantes compartían tres representaciones básicas (un espacio en blanco, un México tradicional y feliz, y un México poco desarrollado e inseguro), construidos con referentes adquiridos frecuentemente en un contexto de inmovili- 
dad, a través de los medios de comunicación, el cine, las webs turísticas, etc. (como también encuentran van der Velde y van Naerssen, 20II). Una vez en el país, aquellas representaciones se tornan más complejas, con la incorporación de referentes locales, la matización o reafirmación de los previos, y la adopción / adaptación de las representaciones locales, construidas en México por la población mexicana.

De ello se desprende que la migración no solo supone un desplazamiento geográfico (en este caso desde España e Italia a México), sino también un “viaje" sociopsicológico (cognitivo, afectivo y mental). Mientras que el primero puede tener un punto de partida y de llegada definidos, cuestión que aquí no se ha abordado, el segundo supone para cada individuo un proceso de duración indeterminada y dependiente de múltiples factores (las representaciones previas, las experiencias vividas, las circunstancias íntimas y relacionales, y las redes de afectividad y cuidado construidas en el país de destino, etc. así como la valoración que se haga de ellas, tal y como también ha mostrado Chacko, 2017), donde no hay una "realidad" definitiva y conclusa a la que llegar ni a la que todos los migrantes desean o aspiran llegar. En este último sentido, por lo tanto, los resultados muestran que las hipótesis de partida de las distancias sociopsicológicas (que las distancias se reducen a medida que aumenta la familiaridad y el contacto) (van Houtum, 2000) no se cumplen para todos los casos, tal y como también señalan Cárdaba y Brändle (2013). Así, mientras que algunos informantes mostraban, a través de las representaciones comunicadas, un acercamiento e incluso adopción de las representaciones de la sociedad local, para otros permanecían y se reafirmaban las representaciones anteriores a la migración, especialmente en cuanto a los estereotipos, lo que confirma su resistencia al cambio a pesar de la familiaridad y contacto. Llegados a este punto, aquí no se pretendía determinar si uno u otro resultado suponen un éxito o fracaso del proceso migratorio, sino poner de manifiesto la complejidad de tal proceso ante diferentes grados de aprensión de la realidad, de conexión afectiva y de comprensión de las convenciones. Sin embargo, para más adelante queda por explorar cómo la experiencia personal de cada migrante en el país de destino puede determinar la reducción o, por el contrario, refuerzo de las distancias sociopsicológicas.

De los resultados obtenidos también se puede concluir que la etapa vital asociada a la condición de migrante (entendida como fenómeno sociológico y psicológico) parece estar caracterizada por un estado de transitoriedad, donde se mezclan lo propio y lo ajeno, y las representaciones del lugar de origen con los del lugar de llegada. Ello se refleja, por ejemplo, en las comparaciones (a veces explícitas y conscientes y otras implícitas e inconscientes) entre el lugar de origen y el de destino, y en la matización y el aumento de la complejidad de las representaciones a partir de la 
experiencia de la "realidad" y de la integración de referentes de las representaciones locales. Este estado de transitoriedad no se define, por lo tanto, por una sustitución o rechazo de las representaciones anteriores, sino que aquellos constituyen la base a partir de la cual construir las posteriores. De ello son ejemplo los casos de reducción de la distancia mental (en la comprensión del "si" que es "no" y de la estructura metageográfica de las diferencias regionales de México), al respecto de los cuales se pueden identificar varias fases, desde la ignorancia, pasando por la incomprensión y frustración y hasta la adopción (donde esta última no es siempre ni para todos los casos la fase final ni deseada). Ahora bien, desde un punto de vista metodológico, debe señalarse que la transitoriedad no se ha determinado individualmente sino colectivamente.

De igual forma, la transitoriedad se manifiesta en las representaciones de los entrevistados que confirmaron y reforzaron las representaciones anteriores, es decir, donde no se produjo una reducción de las distancias. Una muestra de ello es el continuo desplazamiento de las representaciones hacia espacios desconocidos o escasamente experimentados, como es el caso de la búsqueda del "México auténtico" en el centro y sur del país por parte de los migrantes residentes en el norte. Partiendo de una representación previa, aquélla se reconstruye de acuerdo a una representación local, según la cual el Norte no es auténtico ni puro. Un segundo mecanismo identificado consiste en la voluntad de mantener, quizá consciente o inconscientemente, las distancias sociopsicológicas, de evitar "concluir" el proceso de migración e integración. La transitoriedad, la inconclusión, puede entenderse en este sentido como una forma de resistencia ante la pérdida de los referentes propios en detrimento de los ajenos.

Por último, aunque no ha sido lo suficientemente explorado en esta investigación, en paralelo al acercamiento sociopsicológico al país de destino se produce un cierto alejamiento respecto a los países de origen. Ello se manifiesta a través de la incorporación de referentes locales en la (re)producción de las representaciones, que resultan comprensibles para la población local pero no para la de los lugares de origen (como muestra el caso de la metáfora del Suavicrema). Por todo ello se puede concluir que como cualquier "viaje", el sociopsicológico supone a la vez acercarse y alejarse de determinados puntos de referencia, aunque para algunos informantes esos puntos se desplazan también con ellos mientras que, para otros, se mantienen inalterados. 


\section{Bibliografía}

Amin, Ash y Joanne Roberts (eds.) (2008), Community, economic creativity, and organization, Oxford, Oxford University Press.

Ankomah, Paul K., John L. Crompton y Dwayne Baker (1996), "Influence of cognitive distance in vacation choice", Annals of Tourism Research, 23(I), pp. 138-I50. doi.org/I0.1016/0160-7383(95)00054-2.

Anzaldúa, Gloria (1987), Borderlands/La Frontera. The New Mestiza, San Francisco, Aunt Lute Books.

Anzaldúa, Gloria (1993), "Border Arte. Nepantla, el lugar de la frontera”, en Ana Louise Keating (ed.), (2009), The Gloria Anzaldúa Reader, Durham, Duke University Press, pp. I76-I86.

Bender, Steven W. (2012), Run for the Border. Vice and Virtue in U.S.-Mexico Border Crossings, Nueva York, New York University Press.

Cantú, Brenda Olivia et al. (2016), "Percepción que el adolescente residente de Monterrey mantiene sobre el regiomontano", Razón y Palabra, 20(94), pp. IIOO-II2I.

Cárdaba, Miguel Ángel y Gaspar Brändle (2013), "Buscando la inclusión de las minorías en un contexto multicultural. Una revisión teórica del prejuicio y de las estrategias para reducirlo", Papers. Revista de Sociologia, 98(I), pp. 79-I02. dx.doi.org/I0.5565/rev/papers/v98ni.444.

Chacko, Elizabeth (2017), "Exodus, circulation and return. Movements of high-skilled migrants from India in a transnactional era”, en Micheline Van Riemsdijk y Qingfang Wang (eds.), Rethinking international skilled migration, Londres, Routledge, pp. 21I-227.

Cota, Édgar (2007), La representación de la leyenda negra en la frontera norte de México, Turlock, California, Orbis Press.

Cuautle, Alberto (2014), "México el país más surrealista de todos", en Monitor Nacional, io de diciembre de 2014, <http // www.monitornacional.com/mexico-el-pais-mas-surrealista-de-todos/ $>$, (consulta: $31 / 10 / 2016$.

Devadason, Ranji (2010), "Cosmopolitanism, Geographical Imaginaries and Belonging in North London", Urban Studies, 47(14), pp. 2945-2963. doi.org/ı.1177/0042098009360228.

García, José Tomás y Ana Dolores Verdú (2008), "Imaginarios sociales sobre migración: evolución de la autoimagen del inmigrante", Papers: revista de sociologia, 89, pp. 8I-IOI. dx.doi.org/I0.5565/rev/papers/v89no.749. 
Giménez, Gilberto (2007), "La frontera norte como representación y referente cultural en México", Cultura y Representaciones sociales, 2(3), pp. 17-34. Goycoechea, Alba y Franklin Ramírez (2002), "Se fue, ¿a volver? Imaginarios, familia y redes sociales en la migración ecuatoriana a España (1997-2000)", Íconos. Revista de ciencias sociales, I4, pp. 32-45. doi. org/IO.I714I/iconos.14.2002.582.

Hiernaux, Daniel (2002), “Turismo e imaginarios”, en Daniel Hiernaux, Allen Cordero y Luisa van Duynen, Imaginarios sociales y turismo sostenible, San José, Costa Rica, Facultad Latinoamericana de Ciencias Sociales (FLACSO), pp. 7-36.

Houtum, Henk van (1999), "Internationalisation and Mental Borders", Tijdschrift voor economische en sociale geografie, 90(3), pp. 329-335. doi. org/I0.11II/1467-9663.00074.

Houtum, Henk van (2000),"An Overview of European Geographical Research on Borders and Border Regions", Journal of Borderlands Studies, I5(I), pp. 57-83. dx.doi.org/10.1080/08865655.2000.9695542.

Karakayali, Nedim (2009),"Social Distance and Affective Orientations", Sociological Forum, 24(3), pp. 538-562. doi.org/ I0.11II/j.1573-7861.2009.01II9.x. Lewis, Martin W.y Kären Wigen (1997), The Myth of Continents. A Critique of Metageography, Oakland, University of California Press.

Lindón, Alicia y Daniel Hiernaux (dirs.) (2012), Geografías de lo imaginario, Barcelona/México, Anthropos/Universidad Autónoma Metropolitana.

Lussault, Michel (2015) [2007], El hombre espacial. La construcción social del espacio bumano, Buenos Aires, Amorrortu.

Méndez, Eloy y Mario Alberto Velázquez (coords.) (2013), Turismo e imaginarios. Hermosillo, Sonora, El Colegio de Sonora / Instituto Tecnológico y de Estudios Superiores de Monterrey Campus Sonora Norte.

Jodelet, Denise (1984), "Les représentations sociales: phénomènes, concept et théorie", en Serge Moscovici (ed), La psychologie sociale, París, PUF, pp. $357-378$.

Mora, Martín (2002), "La teoría de las representaciones sociales de Serge Moscovici”, Athenea Digital. Revista de pensamiento e investigación social, 2, pp. I-25.

Muscio, Alessandro y Andrea Pozzali (2013), "The effects of cognitive distance in university-industry collaborations: some evidence from Italian universities", The Journal of Technology Transfer, 38(4), pp. 486-508. doi. org/IO.I007/sIO96I-0I2-9262-y. 
Nooteboom, Bart (2000), Learning and Innovation in Organizations and Economies, Oxford, Oxford University Press.

Olmos, Miguel (201I),"Alteridad, etnografía y estereotipos de lo fantástico en la frontera México-Estados Unidos", Cuicuilco, I8(50), pp. 207-227.

Ortiz, Anna y Cristóbal Mendoza (2008), "Vivir (en) la ciudad de México: espacio vivido e imaginarios espaciales de un grupo de migrantes de alta calificación", Latin American Research Review, 43(I), pp. II3-I38.

Peña, Gabriela de la (1998), "Del imaginario internacional de jóvenes regiomontanos", Comunicación y Sociedad, 33, pp. II9-I70.

Prieto, José Manuel (20II), "La consolidación del Monterrey 'imaginario' en el contexto de la globalización:'Macroproyectos' urbanos", Frontera norte, 23(45), pp.163-19I. dx.doi.org/Io.17428/rfn.v23i45.84I.

Rajchenberg, Enrique y Catherine Héau-Lambert (2005), "Las fronteras de la patria”, Estudios Sociológicos, XxIII(67), pp. 239-252.

San Miguel, Pedro (2016), "Muchos Méxicos": Imaginarios históricos sobre México en Estados Unidos, Ciudad de México, Instituto Mora.

Solís, Patricio (2005), "Espacio social y estilos de vida en Monterreyः una evolución crítica de la propuesta de Bourdieu”, en Isabel Jiménez (coord.), Ensayos sobre Pierre Bourdieu y su obra, Ciudad de México, unam / Plaza y Valdés, pp. 313-328.

Tasci, Asli D. A. (2009), "Social Distance. The Missing Link in the Loop of Movies, Destination Image, and Tourist Behavior?", Journal of Travel Research, 47(4), pp. 494-507. doi.org/I0.1177/0047287508326534.

Vargas, Sebastián (2016), "Monumento Estela de Luz: disputas en torno a los usos públicos de la historia en el México del Bi/Centenario", Tempo E Argumento, 8(19), pp. 130-16I. dx.doi.org/10.5965/2175180308192016130.

Velde, Martin van der y Ton van Naerssen (201I), "People, borders, trajectories: an approach to cross-border mobility and immobility in and to the European Union", Area, 43(2), pp. 218-224. doi.org/IO.IIII/j.14754762.2010.00974.x

Yarto, Manuel (20II), "Representación social de los regiomontanos, desde la óptica de las comunidades extranjeras en Monterrey", en Blanca Vázquez y José Juan Olvera (coords.), Procesos comunicativos en la migración. De la escuela a la feria popular, Tijuana / Monterrey, El Colegio de la Frontera Norte / Universidad Regiomontana / Universidad de Monterrey / Instituto Tecnológico y de Estudios Superiores de Monterrey, Pp. 107-I33. 
Zúñiga, Víctor (1992), "El imaginario sobre migración internacional: el deseo de trabajar en Estados Unidos en jefes de hogar del sexo masculino en la Zona Metropolitana de Monterrey. Una aproximación indirecta”, Estudios Fronterizos, 29, pp. 9I-I05.

Zúñiga, Víctor (1998), "Representaciones infantiles de la frontera y del espacio nacional. Materiales para una geografía cultural de la frontera MéxicoEstados Unidos", en: Víctor Zúñiga (coord.), Voces de la frontera. Estudios sobre la dispersión cultural en la frontera México-Estados Unidos, Monterrey: Universidad Autónoma de Nuevo León, pp. 22I-300.

Resumen CURricular Del autor

Xavier Oliveras es Doctor en Geografía por la Universitat Autónoma de Barcelona. Desde 2013 es investigador-profesor en El Colegio de la Frontera Norte (El Colef), en la sede regional de Matamoros. Especializado en espacios transfronterizos y transnacionales, sus temas de interés incluyen la metageografía, la construcción y desarrollo regionales, los regímenes fronterizos y las migraciones. Forma parte del Sistema Nacional de Investigadores (nivel I). Entre sus publicaciones recientes se encuentran:"La resistencia a la refronterización de Estados Unidos: representaciones espaciales en la frontera México-Texas", Aldea Mundo, 2017, núm.43; y“Fiestas transfronterizas y representaciones espaciales en la frontera México-Texas", Economía, Sociedad y Territorio, 2016, núm.50.

Citar como: Oliveras González, Xavier (2018), “Las distancias sociopsicológicas de la migración española e italiana cualificada en México", Iztapalapa. Revista de Ciencias Sociales y Humanidades, núm. 84, año 39, enero-junio de 20I8, ISSN: 2007-9I76; pp. I05-I28. Disponible en <http://revistaiztapalapa. izt.uam.mx/index.php/izt/issue/archive $>$. 